Pure Mathematical Sciences, Vol. 2, 2013, no. 1, 39 - 47

HIKARI Ltd, www.m-hikari.com

\title{
Quasi-Multipliers on Weak \\ Arens Regular Banach Algebras
}

\author{
Marjan Adib \\ Payame Noor University (PNU), Iran \\ pnu.adib@yahoo.com
}

Copyright (c) 2013 Marjan Adib. This is an open access article distributed under the Creative Commons Attribution License, which permits unrestricted use, distribution, and reproduction in any medium, provided the original work is properly cited.

\begin{abstract}
In this paper we define the notion of weak Arens regular Banach algebras and extend the concept of quasi-multipliers to this certain class of Banach algebras. Among other the relationship between Arens regularity of the algebra $A^{* *}$ of a weak Arens regular Banach algebra $A$ and the space $Q M_{r}\left(A^{*}\right)$ of all bilinear and separately continuous right quasimultipliers of $A^{*}$ is investigated. Further, we stablish several properties of the strict and quasi-strict topologies on the $Q M_{r}\left(A^{*}\right)$
\end{abstract}

Mathematics Subject Classification: Primary 47B48; Secondary 46H25

Keywords: Banach algebra, Arens regularity, Quasi-multiplier, multiplier

\section{Introduction}

The notion of a quasi-multiplier is a generalization of the notion of a multiplier on a Banach algebra and was introduced by Akemann and Pedersen [1] for $C^{*}$ algebras. McKennon [14] extended the definition to a general complex Banach algebra $A$ with a bounded approximate identity (b.a.i., for brevity) as follows. A bilinear mapping $m: A \times A \rightarrow A$ is a quasi-multiplier on $A$ if

$$
m(a b, c d)=a m(b, c) d \quad(a, b, c, d \in A) .
$$


For a Banach space $X$, let $X^{*}$ be its topological dual. The pairing between $X$ and $X^{*}$ is denoted by $\langle\cdot, \cdot\rangle$. We always consider $X$ naturally embedded into $X^{* *}$ through the mapping $\pi$, which is given by $\langle\pi(x), \xi\rangle=\langle\xi, x\rangle \quad\left(x \in X, \xi \in X^{*}\right)$.

Let $A$ be a Banach algebra. It is well known that on the second dual $A^{* *}$ there are two algebra multiplications called the first and the second Arens product, respectively. Since in the paper we use mainly the first Arens product, we recall its definition. Let $a \in A, \xi \in A^{*}$, and $F, G \in A^{* *}$ be arbitrary. Then one defines $\xi \cdot a$ and $G \cdot \xi$ as $\langle\xi \cdot a, b\rangle=\langle\xi, a b\rangle$ and $\langle G \cdot \xi, b\rangle=\langle G, \xi \cdot b\rangle$, where $b \in A$ is arbitrary. Now, the first Arens product of $F$ and $G$ is an element $F \circ G$ in $A^{* *}$ which is given by $\langle F \circ G, \xi\rangle=\langle F, G \cdot \xi\rangle$, where $\xi \in A^{*}$ is arbitrary. The second Arens product, which we denote by $\circ^{\prime}$, is defined in a similar way.

The space $A^{* *}$ equipped with the first (or second) Arens product is a Banach algebra and $A$ is a subalgebra of it. It is said that $A$ is Arens regular if the equality $F \circ G=F \circ^{\prime} G$ holds for all $F, G \in A^{* *}$. For example, every $C^{*}$-algebra is Arens regular, see [3]. Note however that $F \circ a=F \circ^{\prime} a$ and $a \circ F=a \circ^{\prime} F$ hold for any $a \in A$ and $F \in A^{* *}$.

The aim of this paper is to present a few new statements on quasi-multipliers of the dual $A^{*}$ of a Banach algebra $A$ whose second dual has a mixed identity.

In our investigation we do not assume Arens regularity, we work on certain Banach algebra which satisfies the weaker condition than Arens regularity and apply our results to $C^{*}$-algebras and to the group algebra of a compact group.

\section{Weak Arens regular Banach algebras}

Definition 2.1 A Banach algebra $A$ is called weak Arens regular if for each $\xi \in A^{*}$ and $F, G \in A^{* *}$ we have

$$
(F \cdot \xi) \cdot G=F \cdot(\xi \cdot G)
$$

Of course, every Arens regular Banach algebra is weak Arens regulsr. However, the class of weak Arens regular Banach algebras is larger. It contains, for instance, every Banach algebra $A$ which is an ideal in its second dual.

Proposition 2.2 Let $A$ be a Banach algebra such that $A \unlhd A^{* *}$. Then $A$ is weak Arens regular.

Proof. Let $F, G \in A^{* *}$ and $\xi \in A^{*}$ be arbitrary. We have

$$
\begin{aligned}
& \langle(F \cdot \xi) \cdot G, a\rangle=\langle\pi(a),(F \cdot \xi) \cdot G\rangle=\left\langle G \circ^{\prime} \pi(a), F \cdot \xi\right\rangle=\left\langle\left(G \circ^{\prime} \pi(a)\right) \circ F, \xi\right\rangle \\
& =\left\langle G \circ^{\prime}(\pi(a) \circ F), \xi\right\rangle=\langle\pi(a) \circ F, \xi \cdot G\rangle=\langle F \cdot(\xi \cdot G), a\rangle \quad(a \in A) .
\end{aligned}
$$


Thus, the class of weak Arens regular Banach algebras is strictly larger than the class of Arens regular algebras.

Proposition 2.3 Let $A$ be a unital Banach algebra. A is weak Arens regular if and only if it is Arens regular.

Proof. Let 1 be the identity for $A$, then then $\pi(1)$ is the identity for $\left(A^{* *}, \circ\right)$ and $\left(A^{* *}, \circ\right)$. Assume that $A$ is weak Arens regular. For arbitrary $F, G \in A^{* *}$ and $\xi \in A^{*}$, one has

$$
\begin{aligned}
\langle F \circ G, \xi\rangle & =\langle F, G \cdot \xi\rangle=\left\langle F \circ^{\prime} \pi(1), G \cdot \xi\right\rangle=\langle\pi(1),(G \cdot \xi) \cdot F\rangle \\
& =\langle\pi(1), G \cdot(\xi \cdot F)\rangle=\langle\pi(1) \circ G, \xi \cdot F\rangle=\langle G, \xi \cdot F\rangle=\left\langle F \circ^{\prime} G, \xi\right\rangle
\end{aligned}
$$

which means that weak Arens regularity implies Arens regularity.

\section{Quasi-multipliers of $A^{*}$ and their properties}

Definition 3.1 $A$ bilinear mapping $m: A^{*} \times A^{* *} \rightarrow A^{*}$ is a right quasimultiplier of $A^{*}$ if

$$
m(F \cdot \xi, G)=F \cdot m(\xi, G) \quad \text { and } \quad m(\xi, G \circ F)=m(\xi, G) \cdot F
$$

hold for arbitrary $\xi \in A^{*}$ and $F, G \in A^{* *}$.

Remark 3.2 Let $Q M_{r}\left(A^{*}\right)$ be the set of all bilinear and separately continuous right quasi-multipliers of $A^{*}$. It is obvious that $Q M_{r}\left(A^{*}\right)$ is a linear space. Moreover, it is a Banach space with respect to the norm

$$
\|m\|=\sup \left\{\|m(\xi, F)\| ; \quad \xi \in A^{*}, F \in A^{* *},\|\xi\| \leq 1,\|F\| \leq 1\right\} .
$$

of $A^{*}$.

Definition 3.3 Let $A$ be a general Banach algebra. Then a map $T: A^{*} \rightarrow A^{*}$ is called a right multiplier of $A^{*}$ if

$$
T(F \cdot \xi)=F \cdot T(\xi)
$$

for all $\xi \in A^{*}, F \in A^{* *}$.

With $M_{r}\left(A^{*}\right)$ we denote the space of all bounded linear right multipliers on $A^{*}$. It is obvious that for each $F \in A^{* *}$ the right multiplication operator $R_{F} \xi=\xi \cdot F$ is a right multiplier on $A^{*}$. 
Theorem 3.4 Let $A$ be a weak Arens regular Banach algebra such that $A^{* *}$ has a mixed identity, then

$$
\rho_{T}(\xi, F)=(T \xi) \cdot F \quad\left(T \in M_{r}\left(A^{*}\right), \xi \in A^{*}, F \in A^{* *}\right)
$$

defines an injective linear map $\rho: M_{r}\left(A^{*}\right) \rightarrow Q M_{r}\left(A^{*}\right)$ with norm $\|\rho\| \leq 1$. Moreover, $\rho$ is onto if $A^{* *}$ has an identity. If $A^{* *}$ has a mixed identity with norm one, then $\rho$ is an isometry.

Proof: Let $T \in M_{r}\left(A^{*}\right)$ be arbitrary. It is obvious that $\rho_{T}$ is a bilinear map from $A^{*} \times A^{* *}$ to $A^{*}$ and that it is bounded with $\|T\|$. For $a \in A, \xi \in A^{*}$, and $F, G \in A^{* *}$, we have

$$
\rho_{T}(F \cdot \xi, G)=T(F \cdot \xi) \cdot G=(F \cdot T \xi) \cdot G=F \cdot(T \xi \cdot G)=F \cdot \rho_{T}(\xi, G)
$$

and

$$
\rho_{T}(\xi, G \circ F)=(T \xi) \cdot(G \circ F)=(T \xi \cdot G) \cdot F=\rho_{T}(\xi, G) \cdot F .
$$

Thus, $\rho_{T} \in Q M_{r}\left(A^{*}\right)$. It follows from the definition that $\rho: M_{r}\left(A^{*}\right) \rightarrow$ $Q M_{r}\left(A^{*}\right)$ is linear. Obviously, $\left\|\rho_{T}\right\| \leq\|T\|$, which gives $\|\rho\| \leq 1$. Let $E \in A^{* *}$ be a mixed identity. If $\rho_{T}=0$, then we have $(T \xi) \cdot E=0$ for every $\xi \in A^{*}$ and consequently $T=0$. Assume that $E$ is an identity for $A^{* *}$. Let $m \in Q M_{r}\left(A^{*}\right)$ be arbitrary. It is easily seen that $T \xi=m(\xi, E)\left(\xi \in A^{*}\right)$ defines a bounded right multiplier of $A^{*}$. Since equalities $\rho_{T}(\xi, F)=(T \xi) \cdot F=m(\xi, E) \cdot F=$ $m(\xi, E \circ F)=m(\xi, F)$ hold for all $\xi \in A^{*}$ and $F \in A^{* *}$ we conclude that $\rho$ is onto.

At the end assume that $E$ is mixed identity for $A^{* *}$ of norm one. Let $T \in M_{r}\left(A^{*}\right)$ and $\varepsilon>0$ be arbitrary. If $\xi \in A^{*}$ is such that $\|\xi\| \leq 1$ and $\|T\|-\varepsilon<\|T \xi\|$, then

$$
\left\|\rho_{T}\right\| \geq\left\|\rho_{T}(\xi, E)\right\|=\|T \xi\|>\|T\|-\varepsilon
$$

Thus, $\rho$ is an isometry.

Remark 3.5 Let $A$ is a weak Arens regular Banach algebra and $A^{* *}$ has an identity. For arbitrary $m_{1}, m_{2} \in Q M_{r}\left(A^{*}\right)$, let $T_{1}, T_{2} \in M_{r}\left(A^{*}\right)$ be uniquely determined multipliers satisfying $m_{1}=\rho_{T_{1}}$ and $m_{2}=\rho_{T_{2}}$. Then

$$
m_{1} \circ_{\rho} m_{2}=\rho_{T_{1}} \circ_{\rho} \rho_{T_{2}}:=\rho_{T_{2} T_{1}}
$$

gives a well defined multiplication. It is easy to see that $Q M_{r}\left(A^{*}\right)$ is a unital Banach algebra. 
Define a map $\psi: A^{* *} \rightarrow Q M_{r}\left(A^{*}\right)$ by $\psi(H)=\rho_{R_{H}}$, where $R_{H}$ is the right multiplication operator on $A^{*}$ determined by $H \in A^{* *}$. Then, for arbitrary $\xi \in A^{*}, F \in A^{* *}$,

$$
\psi(H)(\xi, F)=(\xi \cdot H) \cdot F
$$

Theorem 3.6 Let $A$ be a weak Arens regular Banach algebra and $A^{* *}$ be unital. Assume $A^{*}$ factors on the right. Then $\psi$ is an isomorphism of $A^{* *}$ onto $Q M_{r}\left(A^{*}\right)$.

Proof. We check only the multiplicativity of $\psi$ since the linearity and continuity are evident. Let $H_{1}, H_{2} \in A^{* *}$. By Theorem 3.4, there exist $T_{1}, T_{2} \in M_{r}\left(A^{*}\right)$ such that $\psi\left(H_{1}\right)=\rho_{T_{1}}$ and $\psi\left(H_{2}\right)=\rho_{T_{2}}$. Hence, for arbitrary $\xi \in A^{*}, F \in A^{* *}$, we have

$$
T_{1}(\xi) \cdot F=\left(\xi \cdot H_{1}\right) \cdot F \quad \text { and } \quad T_{2}(\xi) \cdot F=\left(\xi \cdot H_{2}\right) \cdot F .
$$

It follows

$$
\begin{aligned}
\left(\psi\left(H_{1}\right) \circ_{\rho} \psi\left(H_{2}\right)\right)(\xi, F) & =\rho_{T_{2} T_{1}}(\xi, F)=T_{2}\left(T_{1}(\xi)\right) \circ F=T_{1} \xi \cdot\left(H_{2} \circ F\right) \\
& =\xi \cdot\left(H_{1} \circ H_{2} \circ F\right)=\psi\left(H_{1} \circ H_{2}\right)(\xi, F),
\end{aligned}
$$

which means $\psi$ is a homomorphism.

Assume that $\psi(H)=0$ for $H \in A^{* *}$. Since the mapping $\rho$ is one to one $R_{H}=0$. Hence, for each $\xi \in A^{*}$, one has $\xi \circ H=0$. Since, by the assumption, $A^{*}$ factors on the right, we conclude $H=0$. Thus, $\psi$ is one to one. Homomorphism $\psi$ is onto, as well. Namely, if $m \in Q M_{r}\left(A^{*}\right)$, then there exist $T \in M_{r}\left(A^{*}\right)$ such that $m=\rho_{T}=\rho_{R_{T^{*}(E)}}=\psi\left(T^{*}(E)\right)$.

Theorem 3.7 Let $A$ be a weak Arens regular Banach algebra and assume that $A^{* *}$ has an identity $E$. If $A^{* *}$ is Arens regular then $Q M_{r}\left(A^{*}\right)$ is Arens regular.

Proof: Let $\psi$ be as in the proof of Theorem 3.6. Thus, it is an onto homomorphism. Of course, $\psi^{* *}:\left(A^{* *}\right)^{* *} \rightarrow\left(Q M_{r}\left(A^{*}\right)\right)^{* *}$ has the same property, as well. Let $\tilde{F}, \tilde{G} \in\left(Q M_{r}\left(A^{*}\right)\right)^{* *}$. Then there exist $F, G \in\left(A^{* *}\right)^{* *}$ such that $\psi^{* *}(F)=\tilde{F}, \psi^{* *}(G)=\tilde{G}$. Thus,

$$
\tilde{F} \circ \tilde{G}=\psi^{* *}(F) \circ \psi^{* *}(G)=\psi^{* *}(F \circ G)=\psi^{* *}(F \circ G)=\tilde{F} \circ \tilde{G} .
$$

Remark 3.8 Let $A$ be a weak Arens regular Banach algebra. For each $H \in$ $A^{* *}$ and $m \in Q M_{r}\left(A^{*}\right)$ we define

$$
(m * H)(f, G)=m(f, H \circ G) \quad \text { and } \quad(H * m)(f, G)=m(f . H, G)
$$

It is easy to see that $H * m, m * H \in Q M_{r}\left(A^{*}\right)$. 


\section{Strict and quasi-strict topology on $Q M_{r}\left(A^{*}\right)$}

Beside the norm topology there are two other useful topologies on $Q M_{r}\left(A^{*}\right)$. The first is the strict topology $\beta$ which is given by seminorms

$$
m \rightarrow\|m * F\| \quad\left(F \in A^{* *}, m \in Q M_{r}\left(A^{*}\right)\right) .
$$

The second is the quasi-strict topology $\gamma$. It is given by seminorms

$$
m \rightarrow\|m(\xi, F)\| \quad\left(\xi \in A^{*}, F \in A^{* *}, m \in Q M_{r}\left(A^{*}\right)\right) .
$$

Let $\tau$ denote the topology on $Q M_{r}\left(A^{*}\right)$ generated by the norm.

Theorem 4.1 Let $A$ be a weak Arens regular Banach algebra and $A^{* *}$ has a mixed identity. Then $\left(Q M_{r}\left(A^{*}\right), \gamma\right),\left(Q M_{r}\left(A^{*}\right), \tau\right),\left(Q M_{r}\left(A^{*}\right), \beta\right)$ have the same bounded sates.

Proof. Since $\gamma \subseteq \tau$, every $\tau$-bounded set is $\gamma$-bounded. Let $H$ be any $\gamma$-bounded set in $Q M_{r}\left(A^{*}\right)$. Then, for each $\xi \in A^{*}$ and $F \in A^{* *}$, there exist a constant $r=r(\xi, F)>0$ such that

$$
\|m(\xi, F)\| \leq r \text { for all } m \in H .
$$

For each $\xi \in A^{*}$ and $m \in H$, define $M_{\xi}: A^{* *} \rightarrow A^{*}$ by

$$
M_{\xi}(F):=m(\xi, F), F \in A^{* *} .
$$

Then $\mathcal{H}=\left\{M_{\xi}: m \in H\right\} \subseteq C L\left(A^{* *}, A^{*}\right)$. By (1), for any $G \in A^{* *}$,

$$
\left\|M_{\xi}(G)\right\|=\|m(\xi, G)\| \leq r(\xi, G) \text { for all } m \in H
$$

hence $\mathcal{H}$ is pointwise bounded. Then, by the uniform boundedness principle, there exists $c=c(F)>0$ such that

$$
\left\|M_{\xi}\right\| \leq c \text { for all } m \in H .
$$

Consider now the family $P=\left\{p_{m}: m \in H\right\}$ of seminorms on $A^{*}$ defined by

$$
p_{m}(\xi)=\left\|M_{\xi}\right\|=\sup _{\|F\| \leq 1}\left\|M_{\xi}(F)\right\|=\sup _{\|F\| \leq 1}\|m(\xi, F)\|, \quad \xi \in A^{*} .
$$

For each $m \in H, p_{m}$ is continuous on $A^{*}$ since if $\left\{\xi_{n}\right\} \subseteq A^{*}$ with $\xi_{n} \rightarrow \xi_{0}$ in $A^{*}$, then

$$
\left|p_{m}\left(\xi_{n}\right)-p_{m}\left(\xi_{0}\right)\right| \leq p_{m}\left(\xi_{n}-\xi_{0}\right)=\sup _{\|F\| \leq 1}\left\|M_{\xi_{n}-\xi_{0}}(F)\right\|
$$




$$
=\sup _{\|F\| \leq 1}\left\|m\left(\xi_{n}-\xi_{0}, F\right)\right\| \rightarrow 0 .
$$

Then by (2), the family $P$ is pointwise bounded. By [8, p. 142, principle 33.1] there exist a $\operatorname{ball} B\left(\xi_{0}, r\right)=\left\{\xi \in A^{*}:\left\|\xi-\xi_{0}\right\| \leq r\right\}$ and a constant $k_{0}>0$ such that $p_{m}(\xi) \leq k_{0}$ for all $m \in H$ and $\xi \in B\left(\xi_{0}, r\right)$. For fixed $\xi \in A^{*}$ with $\|\xi\| \leq 1$

$$
p_{m}(\xi)=\frac{p_{m}\left(r \xi+\xi_{0}-\xi_{0}\right)}{r} \leq \frac{p_{m}\left(r \xi+\xi_{0}\right)+p_{m}\left(\xi_{0}\right)}{r} \leq \frac{2 k_{0}}{r} .
$$

This implies that

$$
\|m\|=\sup _{\|\xi\| \leq 1,\|F\| \leq 1}\|m(\xi, F)\|=\sup _{\|\xi\| \leq 1} p_{m}(\xi) \leq \frac{2 k_{0}}{r} .
$$

(b) This follows from $(a)$ since $\gamma \subseteq \beta \subseteq \tau$.

\section{Quasi-multipliers of the dual of $L_{1}(G)$}

At the end we consider the group algebra of a compact group $G$. By [19], $L_{1}(G)$ is Arens regular if and only if $G$ is finite. However, since $L_{1}(G)$ is a two-sided ideal in its second dual $([17])$, it is weak Arens regular. Note that the dual $L_{1}(G)^{*}$ can be identified with $L_{\infty}(G)$.

Let $M(G)$ be the convolution algebra of all bounded regular measures on $G$. Recall that the convolution product of $f \in L_{1}(G)$ and $\mu \in M(G)$ is given by

$$
f * \mu(x)=\int_{G} f\left(x y^{-1}\right) d \mu(y) .
$$

Of course, $L_{\infty}(G)$ is a Banach $L_{1}(G)^{* *}$-bimodule. However, the space $L_{\infty}(G)$ has also a natural structure of a Banach $M(G)$-bimodule. The same holds for $L_{\infty}(G)^{*}=L_{1}(G)^{* *}$. We will denote all these module multiplications by $*$.

Proposition 5.1 Let $G$ be a compact group and $A=L_{1}(G)$. Then the equation

$$
\left(\theta_{\mu}(\xi, F):=(\xi * \mu) * F \quad\left(\mu \in M(G), \xi \in L_{\infty}(G), F \in L_{1}(G)^{* *}\right)\right.
$$

defines a linear isomorphism between $M(G)$ and a subspace of $Q M_{r}\left(A^{*}\right)$.

Proof: Note that by the definition of module action $(\xi * \mu) * F=\xi *(\mu * F)$. From this and weak Arens regularity we conclude that $\theta_{\mu} \in Q M_{r}\left(L_{1}(G)^{*}\right)$. Of course, $\theta: M(G) \rightarrow Q M_{r}\left(L_{1}(G)^{*}\right)$ is a bounded linear map. We claim that $\theta$ is injective. Indeed, suppose that $\theta_{\mu}=0$. Then $(\xi * \mu) * F=0$ for all $\xi \in L_{\infty}(G)$ and $F \in\left(L_{\infty}(G)\right)^{*}$. Since $L_{1}(G)$ has a b.a.i. it follows $\xi \circ \mu=0$. In particular, for each $\xi \in C_{0}(G), \xi \circ \mu=0$. Since the measure algebra $M(G)$ is the dual of $C_{0}(G)$ and it has a b.a.i., $\mu=0$, as required. 


\section{References}

[1] C. A. Akemann and G. K. Pedersen, Complications of semicontinuity in $C^{*}$-algebra theory, Duke Math. J. 40 (1973), 785-795.

[2] Z. Argün and K. Rowlands, On quasi-multipliers, Studia Math. 108 (1994), 217-245.

[3] P. Civin and B. Yood, The second conjugate space of a Banach algebra as an algebra, Pacific J. Math. 11 (1961), 847-870.

[4] H. G. Dales, Banach algebras and automatic continuity, London Math. Soc. Monographs, Clarendon press, 2000.

[5] B. Dearden, Quasi-multipliers of Pedersen's ideal, Rocky Mountain J. Math. 22 (1992), 157-163.

[6] R. E. Edwards, Functional Analysis, Theory and Application, Holt, Rinehart and Winston, 1965.

[7] M. Grosser, Quasi-multipliers of the algebra of approximable operators and its duals, Studia Math. 124 (1997), 291-300.

[8] H. G. Heuser Functional analysis, John Wiley \& Sons, Ltd., Chichester, 1982.

[9] M. S. Kassem and K. Rowlands, The quasi-strict topology on the space of quasi-multipliers of a $B^{*}$-algebra, Math. Proc. Cambridge Philos. Soc. 101 (1987), 555-566.

[10] M. Kaneda, Quasi-multipliers and algebrizations of an operator space, J. Funct. Anal. 251 (2007), 346-359.

[11] M. Kaneda and V. I. Paulsen, Quasi-multipliers of operator spaces, J. Funct. Anal. 217 (2004), 347-365.

[12] G. Köthe, Topological Vector Space I, I. New York-Heidelberg-Berlin: Springer, (1969).

[13] H. Lin, The structure of quasi-multipliers of $C^{*}$-algebras, Trans. Amer. Math. Soc. 315 (1987), 147-172.

[14] M. McKennon, Quasi-multipliers, Trans. Amer. Math. Soc. 233 (1977), 105-123. 
[15] A. Ülger, Arens regularity sometimes implies the RNP, Pacific. J. Math 143 (1990), 377-399.

[16] R. Vasudevan and S. Goel, Embedding of quasi-multipliers of a Banach algebra into its second dual, Math. Proc. Cambridge Philos. Soc. 95 (1984), 457-466.

[17] S. Watanabe, A Banach algebra which is an ideal in the second dual space, Sci. Rep. Niigata Univ. Ser. A 11 (1974), 95-101.

[18] R. Yilmaz and K. Rowlands, On orthomorphisms, quasi-orthomorphisms and quasi-multipliers, J. Math. Anal. Appl. 313 (2006), 120-131.

[19] N. Young, The irregularity of multiplication in group algebras, Quart. J. Math. Oxford 24 (1973), 59-62.

Received: September, 2012 